\title{
Breaking up the Proton: An Affair with Dark Forces
}

\author{
Graham D. Kribs" \\ Institute for Fundamental Science and Department of Physics, University of Oregon, Eugene, Oregon 97403, USA \\ David McKeen ${ }^{\dagger}$ and Nirmal Raj® ${ }^{\dagger}$ \\ TRIUMF, 4004 Wesbrook Mall, Vancouver, British Columbia V6T 2A3, Canada
}

(Received 14 August 2020; accepted 8 December 2020; published 7 January 2021)

\begin{abstract}
Deep inelastic scattering of $e^{ \pm}$off protons is sensitive to contributions from "dark photon" exchange. Using HERA data fit to HERA's parton distribution functions (PDFs), we obtain the model-independent bound $\epsilon \lesssim 0.02$ on the kinetic mixing between hypercharge and the dark photon for dark photon masses $\lesssim 10 \mathrm{GeV}$. This slightly improves on the bound obtained from electroweak precision observables. For higher masses, the limit weakens monotonically; $\epsilon \lesssim 1$ for a dark photon mass of $5 \mathrm{TeV}$. Utilizing PDF sum rules, we demonstrate that the effects of the dark photon cannot be (trivially) absorbed into refit PDFs and, in fact, lead to non-Dokshitzer-Gribov-Lipatov-Altarelli-Parisi (Bjorken $x_{B}$-independent) scaling violations that could provide a smoking gun in data. The proposed $e^{ \pm} p$ collider operating at $\sqrt{s}=1.3 \mathrm{TeV}$ (Large Hadron Electron Collider) is anticipated to accumulate $10^{3}$ times the luminosity of HERA, providing substantial improvements in probing the effects of a dark photon: sensitivity to $\epsilon$ well below that probed by electroweak precision data is possible throughout virtually the entire dark photon mass range, as well as being able to probe to much higher dark photon masses, up to $100 \mathrm{TeV}$.
\end{abstract}

DOI: 10.1103/PhysRevLett.126.011801

Introduction.-Are there new gauge interactions in nature? A new, massive Abelian vector boson (dark photon) can, at the renormalizable level, mix kinetically with the standard model hypercharge boson [1]

$$
\mathcal{L} \supset \frac{\epsilon}{2 \cos \theta_{W}} F_{\mu \nu}^{\prime} B^{\mu \nu}
$$

After electroweak symmetry breaking, the dark photon mixes with the standard model (SM) neutral weak boson, whose unmixed versions we denote by $A_{\mu}^{\prime}$ and $\bar{Z}$, respectively. Diagonalizing the kinetic terms and gauge boson masses results in three physical vectors that couple to SM fermions: the massless photon $\gamma$ and the mass eigenstates $Z$ and $A_{D}$.

Numerous searches for $A_{D}$ have been undertaken by directly producing it, in which case the signature depends on its decay mode. In the minimal setup where the only relevant couplings come from Eq. (1), $A_{D}$ decays back into charged SM states, e.g., lepton pairs, offering striking signatures. However, Eq. (1) may serve as our portal to a hidden sector that contains the particle species of the enigmatic dark matter [2,3]. In this case $A_{D}$ might decay invisibly or more nontrivially per the structure of the hidden

Published by the American Physical Society under the terms of the Creative Commons Attribution 4.0 International license. Further distribution of this work must maintain attribution to the author(s) and the published article's title, journal citation, and DOI. Funded by SCOAP ${ }^{3}$. sector. It is therefore desirable to have "decay-agnostic" bounds independent of these details.

In this Letter, we investigate one such decay-agnostic process: deep inelastic scattering (DIS) of $e^{ \pm}$off protons. As seen in the Feynman diagram in Fig. 1, DIS in the presence of kinetic mixing is mediated by the photon, the $Z$, and $A_{D} \cdot A_{D}$ exchange leads to distinct non-DokshitzerGribov-Lipatov-Altarelli-Parisinon (non-DGLAP) scaling violations that may be constrained by existing data and also be the smoking gun in future experiments.

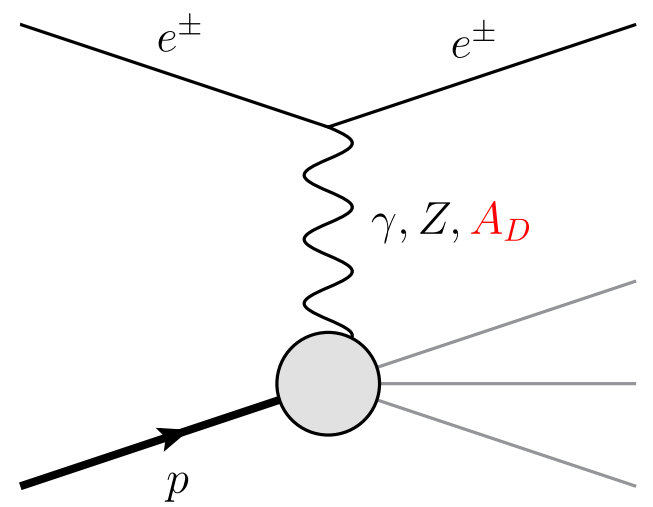

FIG. 1. DIS of $e^{ \pm}$on the proton, mediated by the SM photon and $Z$, and a dark photon arising from kinetic mixing with an Abelian hidden sector. Measurements at HERA and Large Hadron Electron Collider ( $\mathrm{LHeC}$ ) probe the mixing parameter and $A_{D}$ mass relying on no assumptions about the production and decay properties of $A_{D}$. 
Decay-agnostic limits on kinetic mixing were obtained in Refs. [4,5] from electroweak precision observables (EWPOs), driven mainly by the $0.1 \%$ precision $Z$ pole-mass measurements at large electron-positron collider (LEP). The main effect is a shift of the $Z$ mass relative to $m_{W} / \cos \theta_{W}$; using a global fit to EWPOs, a bound of $\epsilon \lesssim$ 0.03 was obtained for $m_{A_{D}} \ll m_{Z}$. We show that DIS measurements at the $e^{ \pm} p$ collider HERA can improve on this bound. With a net luminosity of $1 \mathrm{fb}^{-1}$, HERA achieved $1 \%$ (systematics-limited) precision, however, multiple measurements at this precision give additional statistical power. Decay-agnostic constraints also arise from measurements of the muonic $g-2$, which receives contributions from $A_{D}$-mediated loop amplitudes [6]; these limits, however, weaken considerably for $A_{D}$ masses above the muon mass, becoming negligible above $10 \mathrm{GeV}$. On the other hand, we show that DIS can probe dark photon masses of $10^{4} \mathrm{GeV}$ and beyond.

As discussed above, if assumptions are made about the decay modes of $A_{D}$, additional constraints apply that may be considerably stronger in the region $m_{A_{D}}<10 \mathrm{GeV}$. See Refs. [3,7] for a review of these constraints arising from colliders, beam dump experiments, etc.

Signals of kinetic mixing in deep inelastic scattering.-In this section, we review the basics of DIS (for reviews, see, e.g., [8-10]), incorporating $A_{D}$ exchange. DIS is described by the Lorentz-invariant kinematic variables

$$
Q^{2}=-q^{2}, \quad x_{B}=\frac{Q^{2}}{2 q \cdot p}, \quad y=\frac{q \cdot p}{k \cdot p},
$$

where $q$ is the momentum transfer and $p(k)$ is the incoming proton's (electron's) momentum. The unpolarized neutralcurrent (NC) differential cross section rescaled as a (dimensionless) "reduced cross section" is

$$
\sigma_{\text {red }}^{\mathrm{NC}}=\frac{Q^{4} x_{B}}{2 \pi \alpha^{2}\left[1+(1-y)^{2}\right]} \frac{d^{2} \sigma}{d x_{B} d Q^{2}} .
$$

The cross section can be expressed in terms of the parton distribution functions (PDFs) as per the QCD factorization theorem. In the Quark Parton Model, DIS proceeds via elastic scattering on pointlike quarks and antiquarks, hence their PDFs $f_{q}$ alone contribute and the variable $x_{B}$ becomes the momentum fraction of the proton carried by the parton in the infinite momentum frame; moreover, longitudinal effects are negligible. Neglecting parity-violating effects, $\sigma_{\text {red }}^{\mathrm{NC}}$ is equal to the structure function

$$
\tilde{F}_{2}=\sum_{i, j=\gamma, Z, A_{D}} \kappa_{i} \kappa_{j} F_{2}^{i j}
$$

where $\kappa_{i}=Q^{2} /\left(Q^{2}+M_{V_{i}}^{2}\right)$ accounts for the propagators of vector bosons of mass $M_{V_{i}}$. At leading order [11] in $\alpha_{s}$,

$$
F_{2}^{i j}=\sum_{q} x_{B} f_{q}\left(C_{i, e}^{v} C_{j, e}^{v}+C_{i, e}^{a} C_{j, e}^{a}\right)\left(C_{i, q}^{v} C_{j, q}^{v}+C_{i, q}^{a} C_{j, q}^{a}\right),
$$

where the summation runs over $q=u, \bar{u}, d, \bar{d}, c, \bar{c}$, $s, \bar{s}, b, \bar{b}$, and the vector and axial couplings to fermions (in units of $e=\sqrt{4 \pi \alpha}$ ) are given as follows: For the photon,

$$
\left\{C_{\gamma, e}^{v}, C_{\gamma, u}^{v}, C_{\gamma, d}^{v}\right\}=\left\{-1, \frac{2}{3},-\frac{1}{3}\right\}, \quad C_{\gamma}^{a}=0 .
$$

For the $\bar{Z}$,

$\bar{C}_{Z}^{v} \sin 2 \theta_{W}=T_{3}^{f}-2 q_{f} \sin ^{2} \theta_{W}, \quad \bar{C}_{Z}^{a} \sin 2 \theta_{W}=T_{3}^{f}$,

where $\left\{T_{3}^{e}, T_{3}^{u}, T_{3}^{d}\right\}=\{-1 / 2,1 / 2,-1 / 2\}$ is the weak isospin, $\left\{q_{e}, q_{u}, q_{d}\right\}=\{-1,2 / 3,-1 / 3\} \quad$ is the electric charge, and the Weinberg angle $\sin ^{2} \theta_{W} \simeq$ 0.23127 [14].

We now add the effects of dark photon exchange. First we diagonalize the mixing in Eq. (1) through the field redefinition

$$
B_{\mu} \rightarrow B_{\mu}+\frac{\epsilon}{\cos \theta_{W}} A_{\mu}^{\prime}
$$

and canonically normalize the resulting $A_{\mu}^{\prime}$ kinetic term through the field rescaling

$$
A_{\mu}^{\prime} \rightarrow \frac{A_{\mu}^{\prime}}{\sqrt{1-\epsilon^{2} / \cos ^{2} \theta_{W}}} .
$$

In the $\epsilon \rightarrow \cos \theta_{W}$ limit, this rescaling results in the enhancement of the dark gauge coupling, simultaneously enhancing couplings to SM fermion currents. This, in turn, increases our sensitivity to large $m_{A_{D}}$.

The $\bar{Z}-A^{\prime}$ squared mass matrix becomes

$$
M^{2}=\bar{m}_{\bar{Z}}^{2}\left[\begin{array}{cc}
1 & -\epsilon_{W} \\
-\epsilon_{W} & \epsilon_{W}^{2}+\rho^{2}
\end{array}\right],
$$

where

$$
\begin{aligned}
\epsilon_{W} & =\frac{\epsilon \tan \theta_{W}}{\sqrt{1-\epsilon^{2} / \cos ^{2} \theta_{W}}}, \\
\rho & =\frac{\bar{m}_{A^{\prime}} / \bar{m}_{\bar{Z}}}{\sqrt{1-\epsilon^{2} / \cos ^{2} \theta_{W}}},
\end{aligned}
$$

and the $\bar{Z}-A^{\prime}$ mixing angle is given by 


$$
\begin{aligned}
\tan \alpha= & \frac{1}{2 \epsilon_{W}}\left[1-\epsilon_{W}^{2}-\rho^{2}\right. \\
& \left.-\operatorname{sign}\left(1-\rho^{2}\right) \sqrt{4 \epsilon_{W}^{2}+\left(1-\epsilon_{W}^{2}-\rho^{2}\right)^{2}}\right] .
\end{aligned}
$$

The physical $Z$ couplings are

$C_{Z}^{v}=\left(\cos \alpha-\epsilon_{W} \sin \alpha\right) \bar{C}_{Z}^{v}+\epsilon_{W} \sin \alpha \cot \theta_{W} C_{\gamma}^{v}$,

$C_{Z}^{a}=\left(\cos \alpha-\epsilon_{W} \sin \alpha\right) \bar{C}_{Z}^{a}$,

while those of the physical $A_{D}$ are

$$
\begin{aligned}
& C_{A_{D}}^{v}=-\left(\sin \alpha+\epsilon_{W} \cos \alpha\right) \bar{C}_{Z}^{v}+\epsilon_{W} \cos \alpha \cot \theta_{W} C_{\gamma}^{v}, \\
& C_{A_{D}}^{a}=-\left(\sin \alpha+\epsilon_{W} \cos \alpha\right) \bar{C}_{Z}^{a} .
\end{aligned}
$$

The physical masses are

$$
\begin{aligned}
m_{Z, A_{D}}^{2}= & \frac{m_{\bar{Z}}^{2}}{2}\left[1+\epsilon_{W}^{2}+\rho^{2}\right. \\
& \left. \pm \operatorname{sign}\left(1-\rho^{2}\right) \sqrt{\left(1+\epsilon_{W}^{2}+\rho^{2}\right)^{2}-4 \rho^{2}}\right] .
\end{aligned}
$$

Note that for fixed $\epsilon$ and any value of $\bar{m}_{A^{\prime}} / \bar{m}_{\bar{Z}}$ the difference between the $Z$ and $A_{D}$ masses is always finite, $\left|m_{Z}^{2}-m_{A_{D}}^{2}\right| \geq 2\left|\epsilon_{W}\right| m_{\bar{Z}}^{2}$. This "eigenmass repulsion" is a well-known property of real symmetric matrices, implying that some regions of the $\epsilon-m_{A_{D}}$ plane cannot be realized.

Note that the cross section in Eq. (4) is invariant under $\epsilon \rightarrow-\epsilon$. This arises from requiring $A^{\prime}$ to couple to both quark and lepton currents to be observable at DIS, so that deviations from the SM cross section arise first at $\mathcal{O}\left(\epsilon^{2}\right)$.

For $Q^{2} \ll m_{Z}^{2}$, the short-distance $Z$ exchange is negligible, and $A_{D}$ modifies $\sigma_{\text {red }}^{\mathrm{NC}}$ mainly through its constructive interference with $\gamma$. Thus, it effectively rescales the $\mathrm{SM} \sigma_{\text {red }}^{\mathrm{NC}}$ by $\left[1+\epsilon^{2} Q^{2} /\left(Q^{2}+m_{A_{D}}^{2}\right)\right]^{2}$ in this regime. We illustrate this in Fig. 2 where we plot, for a representative $x_{B}=10^{-2}$, $\sigma_{\text {red }}^{\mathrm{NC}}$ versus $Q$ for $\epsilon=0$ as a band covering $2 \sigma$ uncertainties, and for $\left(\epsilon, m_{A_{D}} / \mathrm{GeV}\right)=(0.1,5),(0.3,5)$, and $(0.3,25)$. Clearly, larger $\epsilon$ values produce larger effects; more subtly, $m_{A_{D}}$ sets the scale in $Q$ above which the effects of $A_{D}$ become significant. Note that the $\epsilon=0.1$ curve lies well outside the SM band, indicating that HERA can probe $\epsilon \ll$ 0.1 with a dataset spanning multiple $x_{B}$.

For $Q \gg m_{Z}$ DIS probes the regime of unbroken electroweak symmetry, where the SM process transpires effectively via massless $B$ exchange. As we will see in the next section, here too the effect of kinetic mixing is to rescale $\sigma_{\text {red }}^{\mathrm{NC}}$ by $\left[1+\epsilon^{2} Q^{2} /\left(Q^{2}+m_{A_{D}}^{2}\right)\right]^{2}$.

HERA constraints and LHeC sensitivities. - To set limits, we use the combined datasets of runs I and II at HERA [12] over the ranges

$$
0.15 \leq Q^{2} / \mathrm{GeV}^{2} \leq 3 \times 10^{4}, \quad 5 \times 10^{-6} \leq x_{B} \leq 0.65 \text {. }
$$

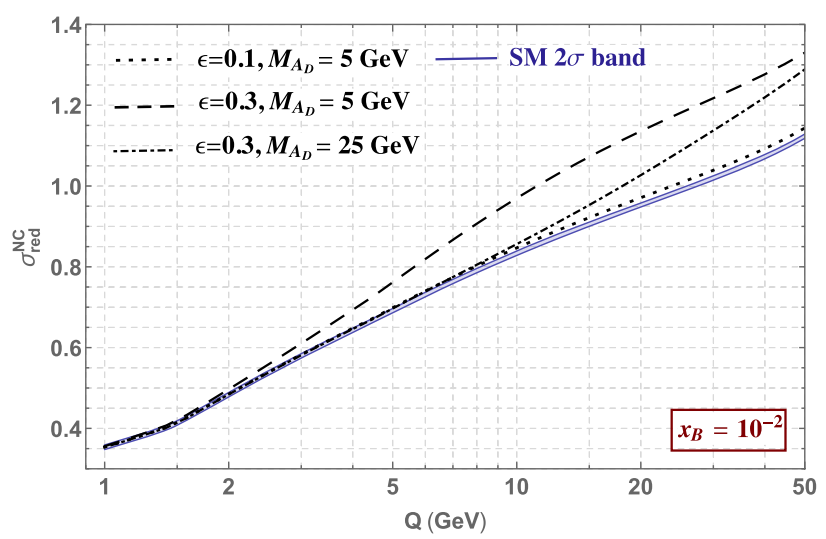

FIG. 2. DIS neutral-current reduced cross sections for a representative $x_{B}=10^{-2}$, as estimated in Eq. (4) using HERAPDF2.0 LO. The blue band covers $2 \sigma$ uncertainties in the SM cross section, obtained by using PDF uncertainties and summing in quadrature the terms in Eq. (4). In this regime, where $Q \ll m_{Z}$, the effects of $Z$ are negligible and $A_{D}$ behaves like a massive photon constructively interfering with $\gamma$, leading to SM cross sections upscaled by $\left[1+\epsilon^{2} Q^{2} /\left(Q^{2}+m_{A_{D}}^{2}\right)\right]^{2}$. Importantly, the cross sections at all $x_{B}$ experience a shift at the same $|Q| \simeq m_{A_{D}}$ that is a non-DGLAP scaling violation, providing a prominent feature of the contribution from $A_{D}$. Already the $\epsilon=0.1$ curve can be visually distinguished from the SM, and when combined with the plethora of measurements at other $x_{B}$ values, it is clear that DIS at HERA can probe the considerably smaller values of $\epsilon \simeq 0.02$, as we show later.

In principle, our constraints must be obtained fitting the HERA data simultaneously to both the $A_{D}$ parameters $\left(\epsilon, m_{A_{D}}\right)$ and the PDFs $f_{q}$. In practice, however, we only fit to $A_{D}$ parameters [15] and use the HERAPDF2.0 LO PDF set derived in Ref. [12] (importing it via ManeParse2.0 [16]).

The bounds we obtain from this simplified approach are, in fact, robust against performing a simultaneous fit. First consider the region $m_{A_{D}} \ll Q_{\min }$, where $Q_{\min }$ is the smallest $Q$ probed at HERA. The $\sigma_{\text {red }}^{\text {NC }}$ are rescaled by $\left(1+\epsilon^{2}\right)^{2}$ with respect to the SM, as discussed before. This implies that the PDFs could absorb this by a simultaneous rescaling of all quark flavors, cf. Eq. (4). However, the normalization of $f_{q}$ is constrained by PDF sum rules. The quark-number sum rules

$$
\int d x_{B}\left[f_{q}\left(x_{B}\right)-f_{\bar{q}}\left(x_{B}\right)\right]= \begin{cases}2, & q=u, \\ 1, & q=d, \\ 0, & q=s, c, b\end{cases}
$$

and the momentum sum rule

$$
\int d x_{B} x_{B}\left[\sum_{q} f_{q}\left(x_{B}\right)+f_{g}\left(x_{B}\right)\right]=1
$$

applied over the HERA $Q$ range are satisfied to $\mathcal{O}\left(10^{-4}\right)$ precision. Using additional data in ranges of $Q^{2}$ outside 
HERA's, such as from beam dumps and hadron colliders, the sum rules can be further constrained once DGLAP evolution is accounted for.

Next, consider the parameter region where $m_{A_{D}}$ is large compared with the momentum exchange for DIS. In this regime, $A_{D}$ can be integrated out, resulting in $\sigma_{\text {red }}^{\mathrm{NC}}$ rescaled by $\left[1+\epsilon^{2} Q^{2} /\left(Q^{2}+m_{A_{D}}^{2}\right)\right]^{2} \simeq 1+2 \epsilon^{2} Q^{2} / m_{A_{D}}^{2}$. The $Q^{2}$ polynomial growth here leads to the largest corrections near $Q^{2} \simeq m_{A_{D}}^{2}$, i.e., near the edge of validity of the effective theory. References $[17,18]$ showed that integrating out new particles modifying quark-lepton interactions, with masses $\gg Q_{\max }$, could be mostly disentangled from the logarithmic scaling of DGLAP evolution. The new physics effects could not be easily "fitted away" into the PDFs.

Finally, for $Q_{\min }<m_{A_{R}}<Q_{\max }$, there is a (smoothed out) step in the rescaled $\sigma_{\text {red }}^{\mathrm{NC}}$ at $Q \simeq m_{A_{D}}$ coming from the factor of $\left[1+\epsilon^{2} Q^{2} /\left(Q^{2}+m_{A_{D}}^{2}\right)\right]^{2}$, illustrated in Fig. 2. Since this step occurs at the same $Q^{2}$ for all $x_{B}$ values, it also does not behave like DGLAP.

To obtain the net uncertainty in $\sigma_{\text {red }}^{\mathrm{NC}}$, we use the HERAPDF uncertainties to sum in quadrature the uncertainties of the terms in Eq. (4). It is these uncertainties that we will use to estimate our limits, as opposed to the errors in the "raw" measurements of the cross sections, since (a) the error covariance matrix is not given, and (b) the PDF fitting procedure accounts for error correlations.

In deriving our bounds, we use the $\left(Q^{2}, x_{B}\right)$ grid used for the HERA run involving $e^{+}$scattering with $\sqrt{s}=318 \mathrm{GeV}$ and $0.5 \mathrm{fb}^{-1}$ luminosity. This grid, containing 485 points, covers most of the $\left(Q^{2}, x_{B}\right)$ used in the other runs involving $e^{ \pm}$scattering at smaller $\sqrt{s}$ and luminosity; although data from all these runs were used for fitting PDFs, we do not use these other grids to avoid oversampling. We derive the 95\% C.L. limit by locating values of $\left(\epsilon, m_{A_{D}}\right)$ for which

$$
\chi^{2}=\sum_{\text {grid }} \frac{\left(\sigma_{\mathrm{red}}^{\mathrm{NC}}-\left.\sigma_{\mathrm{red}}^{\mathrm{NC}}\right|_{\epsilon \rightarrow 0}\right)^{2}}{\left(\delta \sigma_{\mathrm{red}}^{\mathrm{NC}}\right)^{2}}=5.99,
$$

where the summation is over the $\left(Q^{2}, x_{B}\right)$ grid mentioned above. The resulting limits are displayed in Fig. 3. We also show decay-agnostic limits from the E821 $(g-2)_{\mu}$ measurement, requiring $5 \sigma$ deviation from the central value [6], as well as EWPO limits [4]. Our bounds are driven by about 25 data points in the $\left(Q^{2}, x_{B}\right)$ grid where $\sigma_{\text {red }}^{\mathrm{NC}}$ is obtained with a maximum precision of $0.3 \%-0.4 \%$. This is why our limits are (slightly) stronger than EWPOs for $m_{A_{D}} \lesssim 10 \mathrm{GeV} \ll m_{Z}$. Here the observable correction at both LEP $Z$ pole measurements and DIS scales as $\epsilon^{2}$, and while LEP operated at a precision of $0.1 \%$, our bound benefits from 25 independent measurements, effectively diminishing our uncertainty by a statistical factor of $\sqrt{25}$. We also display a hypothetical bound obtained by simply rescaling the $\mathrm{SM} \sigma_{\text {red }}^{\mathrm{NC}}$ by $\left[1+\epsilon^{2} Q^{2} /\left(Q^{2}+m_{A_{D}}^{2}\right)\right]^{2}$, seen to trail the actual bound with amusing proximity. As discussed earlier, such a rescaling amounts to accounting only for $A_{D}$

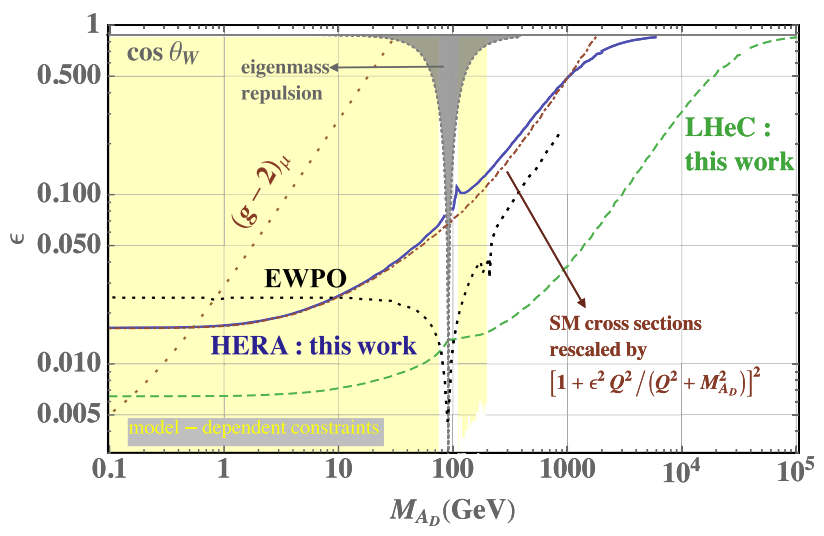

FIG. 3. 95\% C.L. limits from DIS measurements at HERA and future sensitivities at $\mathrm{LHeC}$; similar sensitivities are expected at FCC-eh. For comparison are shown other decay-agnostic limits from EWPO measurements and the muon $g-2$, and, in yellow regions, decay-mode-dependent limits from collider searches. Also shown are hypothetical limits obtained by rescaling SM DIS cross sections by a factor of $\left[1+\epsilon^{2} Q^{2} /\left(Q^{2}+m_{A_{D}}^{2}\right)\right]^{2}$, amounting to accounting only for interference between $A_{D}$ and $B$ exchange. In the gray-shaded region, there is no physical value of $m_{A_{D}}$ in the neighborhood of $m_{Z}=91.1876 \mathrm{GeV}$ due to repulsion of eigenmasses. The change in slope of the HERA and LHeC sensitivity curves at large $\epsilon \gtrsim 0.7$ and $m_{A_{D}} \gtrsim 1 \mathrm{TeV}$ occurs due to a factor of $1 / \sqrt{1-\epsilon^{2} / \cos \theta_{W}^{2}}$ enhancement in the $A_{D}$-fermion coupling. See text for further details.

interference with the $B$ exchange amplitude. Our limits on $\epsilon$ for $m_{A_{D}} \geq 200 \mathrm{GeV}$ agree with ZEUS's limits on contact interactions [18] to within $\mathcal{O}(10) \%$, a nontrivial validation of our treatment above.

We also show the $95 \%$ C.L. future sensitivity of $\mathrm{LHeC}$ [19] derived by using the $\left(Q^{2}, x_{B}\right)$ grid for $e^{+}$scattering over the range

$$
5 \leq Q^{2} / \mathrm{GeV}^{2} \leq 10^{6}, \quad 5 \times 10^{-6} \leq x_{B} \leq 0.8 .
$$

The $\mathrm{LHeC}$ is anticipated to obtain $10^{3}$ times the luminosity of HERA, thus gaining in statistical precision by a factor of about 30 . We are interested in characterizing the maximal sensitivity that $\mathrm{LHeC}$ could achieve with this increased precision, a different objective from obtaining the best-fit PDFs across all datasets. Therefore, to estimate $\mathrm{LHeC}$ sensitivity, we use PDF4LHC15_NNLO_LHEC PDFs fitted to pseudodata [20], but then rescale the fractional uncertainties to match with $2 \times \sqrt{1 / 10^{3}}$ times the fractional uncertainties of HERAPDF2.0 LO, the factor of 2 accounting for systematics potentially dominating the PDF uncertainties. We have checked that rescaling the $Q^{2}$ values of the HERA grid by a factor of $5 / 0.15=10^{6} /\left(3 \times 10^{4}\right)$, the envelopes of smallest uncertainties (as a function of $Q^{2}$ ) for either PDF set are well aligned. We see that LHeC exceeds HERA in the entire $m_{A_{D}}$ range constrained by the latter and, indeed, reaches $m_{A_{D}}$ up to $100 \mathrm{TeV}$ thanks to probing the proton at very high $Q$. Another potential collider future 
circular collider in electron-hadron mode (FCC-eh) is expected to operate at $\sqrt{s}$ that is $2.7 \mathrm{x}$ that of $\mathrm{LHeC}$ with similar luminosities [21], hence we expect it to reach similar couplings and slightly higher $m_{A_{D}}$.

The HERAPDF2.0 LO PDF set is designed to fit solely the HERA DIS data. We used this set not because we believe this is the best description of the quark PDFs, but because this is the most accurate interpolated description of HERA data. Since we are interested in the sensitivity of HERA alone, we believe this is the correct approach. We point out, however, that a more wide-ranging description of PDFs requires "global fits" to HERA combined with beam-dump and hadron collider datasets with complementary ranges of $Q^{2}$ and $x_{B}$. Such PDF determinations contain additional sources of uncertainty [10]: (1) a "tolerance" factor to rescale the goodness of fit so that tensions in fitting multiple datasets may be eased to within $1 \sigma$ uncertainty and (2) parametrization uncertainties introduced by the need to use numerous parameters to fit numerous datasets. The combination of these effects significantly increases the PDF uncertainties. Indeed, we find that, had we used the global PDF set CT18Z [22], our bounds on $\epsilon$ would be weakened by a factor of up to 3 . We do not believe this is a fair characterization of our HERA bounds.

Finally, we note that EWPO sensitivities on $\epsilon$ are expected to improve by $\mathcal{O}(1)$ factors (a factor of $\sim 10$ ) with increased sensitivities provided by future LHC (International linear collider in GigaZ mode) measurements [5].

Discussion.-DIS of $e^{ \pm}$off protons is a sensitive, decaymode-agnostic probe of dark photons up to $100 \mathrm{TeV}$ masses. We find HERA slightly more sensitive than EWPOs for $m_{A_{D}} \lesssim 10 \mathrm{GeV}$. The LHeC could significantly improve the DIS sensitivity, probing $\epsilon$ well below EWPO sensitivity.

It is intriguing to consider discovering a dark photon in DIS. This seems unlikely with HERA data, since EWPOs give stronger constraints for most of the parameter space. The main constraint from EWPOs arises from a shift of $m_{Z}$ versus $m_{W} / \cos \theta_{W}$. It is possible, though unlikely, that other physics in the dark sector could compensate for this apparent contribution to custodial violation and weaken the EWPO bounds. In addition, for $m_{A_{D}} \lesssim 10 \mathrm{GeV}$ where DIS is slightly more sensitive, model-dependent searches, especially from $B$ factories, provide strong constraints. The BABAR Collaboration has searched for $e^{+} e^{-} \rightarrow \gamma A_{D}$ assuming $A_{D}$ decays visibly [23] or invisibly [24], limiting $\epsilon \lesssim 10^{-3}$. Similar searches by LHCb in the $\mu^{+} \mu^{-}$final state constrain $\epsilon \lesssim \mathcal{O}\left(10^{-3}\right)$ for masses below $10 \mathrm{GeV}$ [25] and up to $70 \mathrm{GeV}$ [26,27], while CMS in this channel is sensitive to $m_{A_{D}} \lesssim 200 \mathrm{GeV}$ [28]. These limits, shown in Fig. 3, can potentially be weakened if $A_{D}$ couples to a dark sector with further structure, e.g., [29].

The LHeC's sensitivity is significantly better than EWPOs, enabling direct searches for the non-DGLAP
( $x_{B}$-independent) scaling violation illustrated in Fig. 2, best optimized by simultaneously fitting the PDFs with $A_{D}$ exchange. Nevertheless, we have emphasized that PDF sum rules strongly constrain "fitting away" the effects of $A_{D}$ on PDFs, as borne out by other studies $[17,18]$. The LHeC can also truly discover dark photons in currently unconstrained regions; e.g., we find that the $5 \sigma$ reach in $\epsilon$ for $m_{A_{D}}=1 \mathrm{TeV}(10 \mathrm{TeV})$ is $0.056(0.42)$. Further, our telltale $x_{B}$-independent "step" feature in DIS would motivate new colliders for directly producing the new state and studying its nature in more detail.

In this Letter, we have focused on the effects of a dark photon on DIS, however, this can also be extended to any new force between quarks and leptons, such as mediated by a gauged $\mathrm{U}(1)_{B-L}$ boson, new Higgs sector scalars, or other exotic force carriers. We leave these investigations to future work.

We are grateful to Paddy Fox, Matheus Hostert, David Morrissey, John Ng, Maxim Pospelov, Ennio Salvioni, Michael Spannowsky, Tim Tait, Yue Zhao, and especially Dave Soper for beneficial conversations. The work of G. D. K. is supported in part by the U.S. Department of Energy under Award No. DE-SC0011640. The work of D. M. and N. R. is supported by the Natural Sciences and Engineering Research Council of Canada. TRIUMF receives federal funding via a contribution agreement with the National Research Council Canada. This work was performed in part at the Aspen Center for Physics, which is supported by National Science Foundation Grant No. PHY1607611.

\footnotetext{
*kribs@uoregon.edu

†mckeen@triumf.ca

*nraj@triumf.ca
}

[1] L. Okun, Sov. Phys. JETP 56, 502 (1982); P. Galison and A. Manohar, Phys. Lett. 136B, 279 (1984); B. Holdom, Phys. Lett. 166B, 196 (1986).

[2] M. Pospelov, A. Ritz, and M. B. Voloshin, Phys. Lett. B 662, 53 (2008).

[3] R. Essig et al., arXiv:1311.0029.

[4] A. Hook, E. Izaguirre, and J. G. Wacker, Adv. High Energy Phys. 2011, 859762 (2011).

[5] D. Curtin, R. Essig, S. Gori, and J. Shelton, J. High Energy Phys. 02 (2015) 157.

[6] M. Pospelov, Phys. Rev. D 80, 095002 (2009).

[7] P. Ilten, Y. Soreq, M. Williams, and W. Xue, J. High Energy Phys. 06 (2018) 004; M. Fabbrichesi, E. Gabrielli, and G. Lanfranchi, arXiv:2005.01515.

[8] J. Blumlein, Prog. Part. Nucl. Phys. 69, 28 (2013).

[9] H. Abramowicz et al. (H1 and ZEUS Collaborations), Eur. Phys. J. C 75, 580 (2015).

[10] K. Kovak, P. M. Nadolsky, and D. E. Soper, Rev. Mod. Phys. 92, 045003 (2020).

[11] By using the QCD factorization theorem at next-to-leading order (NLO) and by using NLO PDFs derived by HERA, we 
have checked that treating the problem at NLO gives quite similar results. As discussed in Ref. [12], while fitting PDFs their uncertainties remain roughly the same as one goes to higher orders in $\alpha_{s}$. Other higher-order effects such as multigluon exchange could become important at $Q \lesssim \mathrm{GeV}$ and ow $x_{B}$ [13], but the HERA uncertainties in this region are too large to make any appreciable difference to our constraints.

[12] H1 and Z. Collaborations, Eur. Phys. J. C 75, 580 (2015).

[13] D. E. Soper, M. Spannowsky, C. J. Wallace, and T. M. P. Tait, Phys. Rev. D 90, 115005 (2014).

[14] M. Tanabashi et al. (Particle Data Group), Phys. Rev. D 98, 030001 (2018).

[15] Our procedure can be viewed as estimating the expected sensitivities on our model using a second dataset with the same $\left(Q^{2}, x_{B}\right)$ grid as HERA, with PDFs fitted under the SM null hypothesis. This is precisely what we do when we estimate the sensitivity of $\mathrm{LHeC}$.

[16] D. Clark, E. Godat, and F. Olness, Comput. Phys. Commun. 216, 126 (2017).

[17] S. Carrazza, C. Degrande, S. Iranipour, J. Rojo, and M. Ubiali, Phys. Rev. Lett. 123, 132001 (2019).

[18] H. Abramowicz et al. (ZEUS Collaboration), Phys. Rev. D 99, 092006 (2019).

[19] J. L. A. Fernandez, C. Adolphsen, A. N. Akay, H. Aksakal, J. L. Albacete, S. Alekhin, P. Allport, V. Andreev,
R. B. Appleby, E. Arikan et al., J. Phys. G 39, 075001 (2012).

[20] R. A. Khalek, S. Bailey, J. Gao, L. Harland-Lang, and J. Rojo, SciPost Phys. 7, 051 (2019).

[21] P. Agostini et al. (LHeC and FCC-he Study Group), arXiv:2007.14491.

[22] T.-J. Hou, J. Gao, T. J. Hobbs, K. Xie, S. Dulat, M. Guzzi, J. Huston, P. Nadolsky, J. Pumplin, C. Schmidt, I. Sitiwaldi, D. Stump, and C. P. Yuan, arXiv:1912.10053 [Phys. Rev. D (to be published)].

[23] J. Lees et al. (BABAR Collaboration), Phys. Rev. Lett. 113, 201801 (2014).

[24] J. Lees et al. (BABAR Collaboration), Phys. Rev. Lett. 119, 131804 (2017).

[25] R. Aaij et al. (LHCb Collaboration), J. High Energy Phys. 10 (2020) 156.

[26] R. Aaij et al. (LHCb Collaboration), Phys. Rev. Lett. 124, 041801 (2020).

[27] R. Aaij et al. (LHCb Collaboration), Phys. Rev. Lett. 120, 061801 (2018).

[28] A. M. Sirunyan et al. (CMS Collaboration), Phys. Rev. Lett. 124, 131802 (2020).

[29] M. Baumgart, C. Cheung, J. T. Ruderman, L.-T. Wang, and I. Yavin, J. High Energy Phys. 04 (2009) 014; R. Essig, P. Schuster, and N. Toro, Phys. Rev. D 80, 015003 (2009); A. Katz, E. Salvioni, and B. Shakya, J. High Energy Phys. 10 (2020) 049. 